\title{
Jürgen Stolzenberg, Detlef Döring $\dagger$, Katharina Middell, Hanns-Peter Neumann (Hg.), Briefwechsel zwischen Christian Wolff und Ernst Christoph von Manteuffel: 1738-1748.
}

Christophe Losfeld

\section{(2) OpenEdition}

Journals

Electronic version

URL: https://journals.openedition.org/ifha/11494

DOI: 10.4000/ifha. 11494

ISSN: 2198-8943

Publisher

IFRA - Institut franco-allemand (sciences historiques et sociales)

\section{Electronic reference}

Christophe Losfeld, "Jürgen Stolzenberg, Detlef Döring †, Katharina Middell, Hanns-Peter Neumann (Hg.), Briefwechsel zwischen Christian Wolff und Ernst Christoph von Manteuffel: 1738-1748.", Revue de I'IFHA [Online], Date of review, Online since 13 October 2021, connection on 21 October 2021. URL: http://journals.openedition.org/ifha/11494 ; DOI: https://doi.org/10.4000/ifha.11494

This text was automatically generated on 21 October 2021.

(C)IFHA 


\section{Jürgen Stolzenberg, Detlef Döring $\dagger$, Katharina Middell, Hanns-Peter Neumann (Hg.), Briefwechsel zwischen Christian Wolff und Ernst Christoph von Manteuffel: 1738-1748.}

Christophe Losfeld

\section{REFERENCES}

Jürgen Stolzenberg, Detlef Döring †, Katharina Middell, Hanns-Peter Neumann (Hg.), Briefwechsel zwischen Christian Wolff und Ernst Christoph von Manteuffel: 1738-1748. Historisch-kritische Edition in 3 Bänden, Hildesheim, Zürich, New York (Georg Olms) 2019, 2136 S. (Christian Wolff, Gesammelte Werke, III. Abt.: Materialien und Dokumente, 160), ISBN 978-3-487-15767-2, EUR 744,00. 
Il est deux types d'éditions de correspondance: l'un, relevant d'une entreprise couvrant des décennies, s'attache à la publication d'une correspondance complète, comme c'est le cas de la magistrale édition des lettres de Leibniz (commencée dès 1923) et l'autre, à l'édition des lettres échangées par deux personnes, tâche de moindre envergure, mais non moins intéressante pour le lecteur, comme en témoigne l'édition historique et critique des lettres de Christian Wolff et Ernst Christoph v. Manteuffel entre 1738 et 1748 . Dans un cas comme dans l'autre, disposer d'un corpus sinon complet, du moins conséquent ${ }^{1}$, est une chance inouïe, qu'ont bien mise à profit Detlef Döring, à l'origine de cette

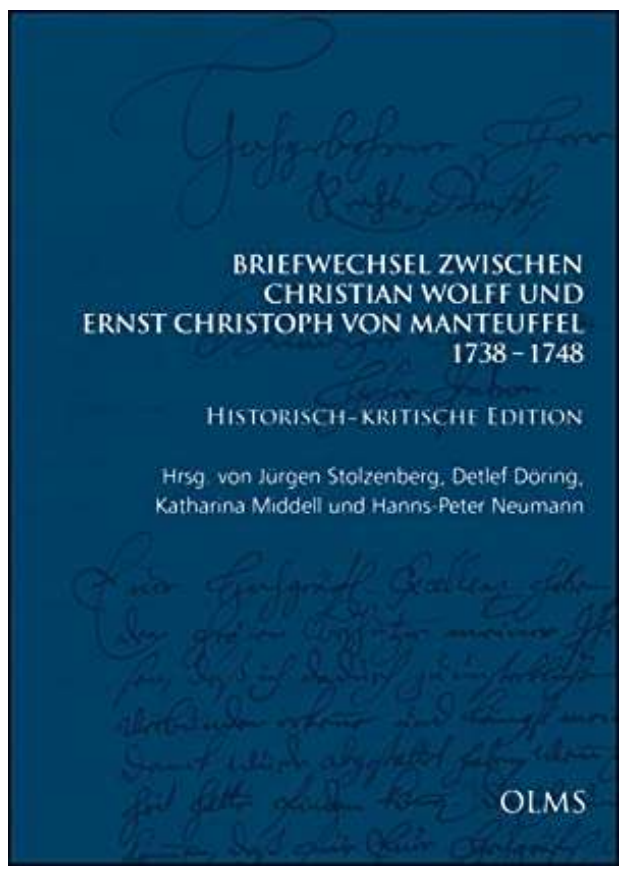
édition, ainsi que Jürgen Stolzenberg, Katharina Middell et Hanns-Peter Neumann qui l'ont poursuivie et achevée après son décès.

Pour ce qui est de Christian Wolff, cette chance est d'autant plus grande qu'une partie non négligeable de la vaste correspondance qu'il entretenait en éminent représentant de la République des lettres a disparu. Cette édition en trois volumes reposant sur un fonds d'archives déposé à la Bibliothèque universitaire de Leipzig rassemble 482 lettres ${ }^{2}$ - soit plus de la moitié des lettres de Wolff qui ont été conservées -et 57 pièces ajoutées en appendice (qu'il s'agisse de lettres échangées avec d'autres personnalités - par exemple Jean Henri Samuel Formey, Jacob Vernet - ou de textes de d'autres érudits, comme Johann Friedrich Wilhelm Jerusalem ou Johann Heinrich Gottlob Justi, que Manteuffel, souvent, avaient joints à ses lettres et qui éclairent la correspondance proprement dite).

Cette correspondance s'étend de mai 1738 à novembre 1748 et couvre donc la période allant de la fin des années que Wolff passa à Marburg, après avoir été chassé de Halle en 1723, aux années qu'il vécut dans cette dernière ville, après avoir été réhabilité en 1740 et où il connut alors la consécration. Et elle ne s'achève pratiquement qu'à la mort de Manteuffel, le 30 janvier 1749 (la dernière lettre est datée du 5 novembre 1748).

L'intérêt de cette correspondance est indissociable de la position sociale occupée par le correspondant de Wolff, et que laisse percevoir l'entrée des lettres de ce dernier. ${ }^{3}$ Manteuffel (1676-1749), issu d'une famille noble de Poméranie fit, après des études de droit et un bref épisode prussien, une brillante carrière politique au service de la Saxe puis de la Saxe polonaise - Frédéric-Auguste $\mathrm{I}^{\mathrm{er}}$ de Saxe était devenu roi de Pologne en 1696 - dont il dirigea même la politique extérieure entre 1728 et 1730 . Après s'être retiré des affaires, il séjourna d'abord dans sa maison de plaisance en Poméranie ultérieure, puis à Berlin où il partagea son temps entre des tâches diplomatiques et la " mission » tant de diffusion des thèses de Wolff que de sa réhabilitation. Chassé de la capitale prussienne en 1740, Manteuffel, véritable " mécène des Lumières ${ }^{4}$ et " le plus grand défenseur de la philosophie » wolffienne ${ }^{5}$, continue, à partir de Leipzig, à 
s'attacher à la propager à la faveur aussi de la "Société des amis de la vérité » ("Societas Alethophilorum ») qu'il avait fondée, dès 1736, et qui rassemblait des partisans des lumières, à l'instar des théologiens Johann Gustav Reinbeck (1683-1741) ou Johann Heinrich Samuel Formey (1711-1797). La place éminente occupée par Manteuffel à l'intersection de la République des lettres et de la politique est un des facteurs expliquant l'intérêt scientifique immense que représente l'édition des lettres qu'il a échangées avec Wolff et qui permet d'approfondir ou de préciser maints des résultats auxquels étaient parvenus les innombrables travaux de recherche sur Wolff publiés à ce jour.

En effet, le lecteur découvre au fil de la correspondance, outre les témoignages d'une amitié 6 que n'obscurcissent que de rares tensions (31), une quantité innombrable de remarques concernant les événements politiques importants de l'époque, comme la Seconde guerre de Silésie (1744-1745), mais aussi la Politique, lorsque Manteuffel, par exemple, s'en prend à ces Princes d'Allemagne dont «fourmille » l'Allemagne et qui, pour la plupart, «sont les oprobres et les fleaux de la Société, dont ils devroient être l'ornement et le soûtien" (4, p. 19) ou qu'il reproche à «la pluspart des souverains d'aujourd'hui [...] de gouverner leurs Etats arbitrairement, et sans conseils. C'est ce qui leur fait ignorer et mépriser la Vérité, et les règles du bon-sens » (208, p. 189). Et en 1748 encore, Manteuffel qui n'est lui-même pas libre de certains des préjugés de son temps - il dénonce, par exemple, la «Vanité » et la «Légèreté impardonnables » du Maréchal de Belle-Ile, deux " qualitéz qui lui sont naturelles, comme à toute sa nation » (201, p. 170) - approuve sans réserve ce que lui écrit Wolff « au sujet de la fausse idée que la pluspart des grans de la terre se font de la grandeur, de la gloire, et de la réputation" (466, p. 455). Les deux correspondants n'ignorent pas, en effet, les conséquences de l'ambition ni les suites néfastes de la guerre sur les Arts et les sciences (231, p. 253 : «Pour les Muses surtout, il est affligeant que le feu de la guerre gagne toujours plus en vigueur et que ses flammes ne cessent de se propager »- «Es ist aber vor die Musen betrübt, daß der Kriegsfeuer noch immer stärcker wird und die Flamme weiter um sich greiffet »).

Que la guerre nuise aux sciences est une catastrophe aux yeux de cet érudit et savant polyvalent qu'est Wolff, comme en témoignent de grands pans de la correspondance. Son intérêt pour toutes les recherches et découvertes scientifiques, à une époque où les frontières disciplinaires n'existent pas encore, laissent pantois le lecteur. Au gré des informations ou des textes (37) que lui transmet Manteuffel, Wolff évoque la géographie, l'astronomie (214), en particulier à l'occasion de l'expédition menée par Pierre Louis Maureau de Maupertuis en Laponie afin de mesurer la planète (176) - le savant français, membre à partir de 1740 de l'Académie de Berlin, et qui offrira à Wolff son Astronomie nautique (206), est un personnage récurrent de la correspondance. En biologie, Wolff fait état de questions de zoologie, lorsqu'il revient sur les polypes étudiés par Abraham Tremblay (196) ou de botanique $(173,237)$; il aborde des questions de physique, exposant la méthode expérimentale qu'il applique (151), s'interrogeant sur la fragilité du verre $(143,155)$ ou sur la question du gel sur les fenêtres (162) et, ce qui constitue par moments un véritable fil rouge dans ses lettres, le problème de l'électricité $\left(124,154,157,167^{7}\right)$. Que l'électricité joue ici un rôle si important tient évidemment à sa fonction pour comprendre les phénomènes naturels (175), mais sans doute aussi à l'importance qu'elle revêt dans les thèses newtonniennes (159). Wolff se penche aussi sur des questions de chimie, comme celle de la poudre (159) ou de l'essence de térébenthine (194). Il s'intéresse principalement aux vertus 
thérapeutiques de ces dernières, ce qui ne tient pas, assurément, à un goût purement théorique pour les questions médicales $(172,182)$, et doit, bien davantage, être rapporté à son désir de traiter les maladies dont il souffre $(185,187)$ : refusant, pour se soigner, des moyens aussi radicaux que la saignée (477), il préfère laisser agir la nature ( « Ich laße gerne die Natur gewehren » 343, p 84) et recommande de recourir tantôt à des eaux médicinales (476), tantôt à des médicaments dont il rédige lui-même la recette (178) et il se montre soucieux de diététique (159), un aspect resté dans l'ombre à ce jour.

Wolff, bien sûr, n'envisage pas uniquement des questions relevant des sciences exactes et se livre, de manière réitérée, à des réflexions psychologiques sur la question des fantômes $(160,320)$, réflexions qui constituent une contribution intéressante et un apport nouveau tant à un vif débat au XVIIIe siècle qu'à un sujet qui, depuis quelques années, retient l'attention de la critique. ${ }^{8} \mathrm{~A}$ une époque où les questions religieuses sont prédominantes encore, il n'y a, certes, guère lieu de s'étonner de la place qu'elles occupent dans la correspondance. L'intérêt de cette dernière est, pourtant, de mettre en lumière des aspects originaux de la pensée wolffienne concernant la création et les caractéristiques des animaux qui en découlent (338) ou encore le péché originel (341). Défenseur de la religion protestante (310) dans la lutte contre le matérialisme propagé, par exemple dans l'Homme machine de La Mettrie, paru en 1747 (et dont la paternité, en un premier temps, est attribuée faussement au Marquis d'Argens tant par Wolff que par Manteuffel, 416 sqq.), Wolff n'hésite cependant pas à remettre en question les thèses luthériennes, dès lors qu'il les juge contraires " aux premiers principes de la science " (« mit den ersten Gründen der Grundwißenschaft streitet » 341, p. 78).

Une part non négligeable de la correspondance est bien sûr consacrée aux travaux juridiques de Wolff, qu'il s'agisse de ceux prévus, en cours ou achevés $(18,356)$ et les nombreuses remarques qui parsèment les volumes mettent le lecteur à même de suivre, par exemple, la gestation et l'évolution du Jus Naturae, évolution qui montre bien combien d'autres publications, de nouvelles informations ou, simplement, des questions posées 9 peuvent en infléchir le cours (315)

Les trois tomes de la correspondance, au-delà des innombrables informations qu'ils livrent sur les sciences à l'époque des Lumières, sont aussi une source précieuse sur le mode de fonctionnement de ses institutions scientifiques. Cela vaut pour des points de détail - comme le souci de réduire le nombre de cours pour pouvoir se concentrer sur la recherche (3), les démarches nécessaires pour accéder à un poste et la déception de ne pas être nommé - autant de phénomènes qui n'ont rien perdu de leur actualité ${ }^{10}-$, et non moins pour des questions essentielles. De nombreuses lettres rendent sensible, de fait, la collusion du politique et des sciences alors, que ce soit lors du scandale opposant Gotthilf August Francke et le Roi de Prusse à propos des théâtres (210), à l'occasion de la confiscation d'un traité sur le pêché originel (244) sans parler, bien sûr, des déboires de Wolff lui-même à Halle et de son installation à Marburg (40).

Cela ne veut point dire que les relations entre les représentants pouvoir politique et les universitaires soient toujours déclinées sur le mode du conflit, car les uns et les autres peuvent, bien au contraire, en tirer profit. Les savants peuvent, par exemple, profiter de l'influence des Grands pour entrer en relation avec leurs pairs, comme l'illustre l'idée de Wolff d'essayer d'entrer en contact avec Voltaire, après que le prince héritier l'a cité dans une lettre envoyée au philosophe français (30), ou pour gagner ou regagner en influence : Sensibiliser, lors de conversations particulières, la reine de Prusse à la 
philosophie wolffienne peut, ainsi, participer des efforts déployés pour réhabiliter Wolff $(23,28)^{11}$. Et la correspondance de Manteuffel et Wolff illustre également le rôle de la dédicace d'un ouvrage pour gagner les faveurs d'un prince (16, 17, 240 et 351). Le caractère positif des liens entre le politique et la science apparaît aussi lorsqu'un souverain émet le vœu de faire nommer un savant dans l'académie de son pays, moyen, bien sûr, de "donner du lustre à l'Academie » (comme le dit Manteuffel lui-même à propos du désir du roi du Danemark d'inviter Wolff, 317, p.12) et d'honorer celui-là, tout en permettant de renforcer les liens au sein de la République des lettres et de diffuser les idées nouvelles.

Le rôle, bien connu déjà, des académies dans la diffusion des Lumières explique, en retour, le scandale que provoque l'attribution des prix qu'elles décernent à des auteurs qui, selon les représentants éclairés de la République des lettres ne le mériteraient pas, comme cela se produit lorsque Johann Heinrich Gottlob Justi (1720-1771) remporte le prix de l'Académie de Berlin pour un texte concernant les monades. ${ }^{12}$ Wolff se montre outré, reproche au jury son incompétence (328 sqq.) et renouvelle plusieurs fois sa critique dans ses lettres (344), de même qu'il s'en prend vertement aux académies approuvant des textes qui ne sont que « gribouillage» («Schmierereyen» 362, p. 143). Wolff, au nom de la dignité qui devrait caractériser les érudits et les savants, refuse, cependant, de se laisser entraîner dans des campagnes de diffamation (195) ou d'entrer en polémique avec un Justi (362) qu'il incite à la modération (380), arguant qu'il n'est pas convenable « de transformer un journal en théatre de controverses » («daß man ein Journal zum Theatro einer Controvers machen wil» 362, p. 143), de même qu'il critique les intrigues de certains universitaires visant à entacher la réputation de certains de leurs collègues (3), ce qui témoigne, a contrario, de la vivacité des débats au sein de la République des Lettres.

Il est vrai que différents courants s'y livrent à une lutte d'influence: Wolff et ses partisans s'efforcent ainsi de lutter tant contre le matérialisme (30) et certaines thèses philosophiques que certains professeurs empruntent «chez certains Anglais et chez Voltaire » $\left(56\right.$, p. 240 : «bey einigen Engelländern und dem de Voltaire ») ${ }^{13}$, que le newtonisme qui «nuit énormément aux sciences» («thut den Wißenschaften gewaltigen Schaden» 458, p.436). Pour s'opposer à l'influence de telles théories néfastes (38), Wolff et ses partisans opposent s'efforcent de diffuser par plusieurs canaux la philosophie wolffienne, que ce soit dans des cercles privés où se rencontrent des érudits qui s'adonnent à des « escarmouches philosophiques» (262, p. 355), par les liens, on l'a relevé déjà, avec le Grands, ou l'extension d'un réseau (202) de gens adhérant au wolffianisme... Ces différents moyens visent à la propagation des thèses de Wolff dans toute l'Europe : en Angleterre - où les conditions sont pourtant défavorables $(325,240)$-, en Pologne (240), en Hollande (251), ou encore au Danemark (351) et passent par des traductions des œuvres de Wolff ou leur adaptation dans des genres susceptibles d'atteindre un public plus large, comme le roman la Belle Wolfienne de Jean Henri Samuel Formey $(242,256)^{14}$ qu'approuve Wolff (la traduction de certaines des œuvres de Wolff est critiquée, en revanche, 54).

Ces quelques indications qui ne prétendent en rien donner une idée de toutes les facettes thématiques de cette correspondance suffisent à laisser deviner l'intérêt scientifique qu'en représente l'édition. Tous les aspects qu'elle reflète n'étaient, évidemment, pas méconnus ${ }^{15}$, mais elle les rassemble, permettant au lecteur 
d'embrasser en une vue panoramique le Siècle des Lumières et de percevoir mieux la personnalité des deux correspondants.

Que sa lecture de ces trois volumes soit si fructueuse est indéniablement dû à l'excellence de la qualité de l'édition. Outre les qualités haptiques et optiques de l'ouvrage, en parfaite conformité avec les principes de la maison d'édition olms, les éditeurs ont pris un certain nombre de décisions tout à fait heureuses. Après une introduction retraçant la naissance de cette entreprise (I, pp. xxvii-xxxiv), ils dressent ainsi un bref tableau des principaux sujets abordés par Wolff et Manteuffel, dont ils dépeignent ensuite l'horizon biographique (xxxivl-vii)

Dans le corps du texte proprement dit, ils indiquent précisément la provenance des lettres ayant été conservées - le méticuleux travail philologique leur permettant, parfois, d'élucider la date de rédaction (voir 205) ou de dresser une liste très complète des lettres ayant disparu (I, lxii-lxiv). La transcription de chaque lettre est toujours précédée d'un court résumé de son contenu, ce qui est fort appréciable pour les lecteurs, même spécialistes du XVIIIe siècle - à qui s'adresse cette édition -, dont les connaissances linguistiques ne suffiraient pas à lire avec la fluidité nécessaire le latin (que les deux scripteurs n'utilisent que ponctuellement), le français de l'époque, que privilégie Manteuffel, ou même la langue allemande, parfois épineuse, de l'aube des Lumières. Le texte est commenté dans une double perspective : d'une part les variantes sont indiquées, les abréviations décryptées, pour autant qu'elles ne figurent pas dans la liste des abréviations principales donnée au début de chaque volume, et les citations latines traduites que les deux scripteurs essaiment. D'autre part, les commentaires donnent toutes les informations nécessaires à une véritable compréhension de la lettre en indiquant minutieusement les sources citées implicitement par l'un des correspondants (qu'il s'agisse du titre des ouvrages mentionnés, voire de passages précis des œuvres de Wolff ou d'un autre auteur) ${ }^{16}$, en précisant les événements auxquels renvoient Wolff et Manteuffel, ou en donnant des indications biographiques sur les personnes évoquées. Les personnages mentionnés de manière récurrente dans les lettres font l'objet, de plus de brèves biographies à la fin du troisième volume, - ce qui évite de surcharger un appareil critique déjà fort riche, ainsi que les redondances. Les commentaires, précis, exhaustifs et se fondant sur une large bibliographie de sources et de littérature critique indiquée à l'orée de chaque volume, éclairent largement des lettres. Et ces commentaires loin de rendre superflue la lecture des lettres, incitent bien davantage à s'y plonger, ce qui n'est la moindre qualité de cette édition. Et il ne faut omettre de mentionner ni le système de concordance entre les lettres que proposent les éditeurs, et qui permettent au lecteur s'intéressant à des sujets particuliers d'en suivre mieux le fil, ni l'heureuse initiative qu'ils ont prise de mettre à la disposition du public une version en ligne de l'édition (sans commentaires) -https://slub.qucosa.de/landing-page/?

tx_dlf[id]=https\%3A\%2F\%2Fslub.qucosa.de\%2Fapi\%2Fqucosa\%253A2854\%2Fmets - qui rend possible une recherche ciblée.

Jürgen Stolzenberg, l'un des éditeurs, est aussi l'un des fondateurs de la «ChristianWolff-Gesellschaft für die Philosophie der Aufklärung » qui a été fondée en 2017 afin de faire connaître celui qu'on appelait, en dépit des innombrables travaux qui lui ont été consacrés en France comme en Allemagne depuis le siècle dernier, "le philosophe presque oublié des Lumières $»{ }^{17}$ Cette excellente édition de la correspondance entre 
Wolff et Manteuffel est, à n'en pas douter, une date clef dans la redécouverte de cet érudit.

\section{NOTES}

1. Pas moins de 94 lettres de la correspondance entre Wolff et Manteuffel ont pareillement disparu (voir la liste établie par les éditeurs, T. I, pp.lxii sqq.)

2. Tome I : lettres 1-150 ; tome II : 151-314; tome III 315-488.

3. Il commence généralement ses lettres par « Hochgebohrner Reichs=Graffe / Gnädiger Herr / Hoher Patron» («Comte d'Empire de haute naissance / Seigneur [miséricordieux] / Grand protecteur.

4. Tel est le titre de la thèse de Johannes Bronisch qui a décrit et analysé minutieusement le corpus publié ici (Der Mäzen der Aufklärung: Ernst Christoph von Manteuffel und das Netzwerk des Wolffianismus. Berlin : De Gruyter 2010, [=Frühe Neuzeit, 147, Band 147].

5. L'expression est de Wolff lui-même (1, p.5). Il est à noter que Manteuffel s'avère un intermédiaire précieux non seulement pour la diffusion des thèses wolffiennes, mais dans des questions aussi banales, du moins pour le lecteur actuel, que, par exemple, l'achat d'une nouvelle maison, puisque Wolff utilise les influences dont dispose Manteuffel pour parfaire le contrat (333).

Pour des raisons pratiques, le renvoi sera fait au numéro des lettres, auquel on adjoindra, dans le cas de citations, celui de la page. Il va de soi que les renvois, loin de prétendre à l'exhaustivité, n'ont que valeur d'exemple.

6. Manteuffel accueillera par exemple plusieurs semaines le fils de Wolff sous son toit (471).

7. En physique, Wolff souvent reste favorable aux thèses de Descartes (397).

8. Voir Caroline Callard: Le temps des fantômes. Sprectralités de l'âge moderne (XVIe-XVIIe). Paris 2019, Yvonne Wübben: Gespenster und Gelehrte: Die ästhetische Lehrprosa Georg Friedrichs Meiers (1718-1777), Tübingen 2007.

9. C'est ainsi, par exemple, que Manteuffel invite Wolff à réfléchir à la nécessité de continuer à distinguer le droit des gens et celui de la nature (126).

10. Obtenir un poste dans une université peut évidemment contribuer aussi à pouvoir propager les Lumières $(29,41)$.

11. Les lettres publiées ici permettent d'éclairer sous un jour nouveau les multiples canaux utilisés pour, justement, parvenir à la réhabilitation de Wolff : la carrière qui lui proposée à Francfort sur le Main est perçue comme un premier pas vers la réhabilitation complète (27), à laquelle œuvrent également les épigones de Wolff (39) et, en particulier Manteuffel, bien évidemment (28) qui, en parfait diplomate, use de son crédit auprès du roi pour plaider en faveur de Wolff tout en invitant à la prudence (32). Ces efforts semblent couronnés de succès: Dès octobre 1739, un bruit court que Wolff repartira pour Halle en 1740 (42) - ce que ce dernier dément (45)- et on lui soumet même la proposition de devenir vice-chancelier de l'Université (43). Après plusieurs péripéties que les lettres permettent également de suivre avec précision, Wolff fera un retour triomphal à Halle le 6 décembre 1740 .

12. Les commentaires apportés aux lettres sont, dans l'édition, toujours de grande qualité. Dans le cas du débat sur les monades, ils profitent évidemment de ce que Hanns-Peter Neumann, l'un des éditeurs est un spécialiste avéré de la question, comme en témoigne son ouvrage : Monaden im 
Diskurs. Monas, Monaden, Monadologien (1600 bis 1770), Stuttgart: Franz Steiner, 2013 [= Studia Leibnitiana Supplementa, 37].

13. L'image de Voltaire donnée par la correspondance est ambivalente: Manteuffel se montre beaucoup plus critique à l'encontre du philosophe français (auquel il reproche sa «Philautie demesurèe » et le fait " qu'il donne, à corps perdu, dans le Materialisme et le Scepticisme » $(33$, p. 136) qu'un Wolff qui, partageant tout d'abord cette vision critique (35) change d'opinion après qu'il a lu les remarques positives que Voltaire avait faites faites sur lui dans sa correspondance avec Frédéric (211 et 219).

14. Wolff se réjouit des efforts de théologiens comme Heinrich Wilhelm Bernsau qui s'efforce d'appliquer à la théologie les thèses de $\mathrm{W}$ en dépit de grandes réticences (481).

15. Pour d'évidentes raisons, nous renonçons ici à renvoyer pour chacun des sujets évoqués, à des articles ou monographies déjà existantes - que les lettres de la correspondance complètent bien souvent - ou encore avec les entrées, par exemple, du récent Handbuch Christian Wolff édité par Robert Theis et Alexander Aichele (Wiesbaden : Springer 2018).

16. L'impression, en appendice, de 57 textes trouvés dans les fonds d'archives participe de cette volonté d'éclairer au mieux la correspondance.

17. https://www.campus-halensis.de/artikel/der-fast-vergessene-philosoph-christian-wolffgesellschaft-gegruendet/ (consulté le 10/09/2021).

INDEX

Chronological index: Période moderne

Subjects: Histoire des idées

\section{AUTHOR}

CHRISTOPHE LOSFELD

Institut für Romanistik Martin-Luther-Universität Halle-Wittenberg 